\title{
Evaluación clínica de gel a base de extracto hidroalcohólico de Pelargonium robertianum L. (geranio) para el tratamiento de las hemorroides
}

\section{Clinical Evaluation of Gel Based on Hydroalcoholic Extract of Pelargonium robertianum L. (geranium) for the treatment of hemorrhoids}

\author{
Carlos Alejandro Bell Cortez*
}

http://dx.doi.org/10.21503/CienciayDesarrollo.2010.v12.01

\section{RESUMEN}

Se evaluó el efecto sobre las hemorroides de un gel con $1 \%$ de extracto hidroalcohólico de hojas de Pelargonium robertianum $L$. (geranio), en 85 voluntarios de ambos sexos con edades comprendidas entre 20 y 50 años, todos residentes en la ciudad de Lima (Perú). El ensayo, previo consentimiento informado, mostró tener efecto en el proceso desinflamatorio de las hemorroides. El 74,3 \% fue evaluado como excelente, el 22,8 \% como bueno y el 2,9 \% como regular. Durante el ensayo no se reportaron reacciones alérgicas, y el producto fue bien tolerado por los pacientes. Los resultados recién se observaron luego de la primera semana de tratamiento. Debido a su origen natural y a su bajo costo, el presente gel puede constituirse en una buena alternativa para el tratamiento de hemorroides.

Palabras clave: Pelargonium robertianum L., hemorroides, estudio clinico, dermatologia.

\section{ABSTRACT}

The effect of a gel with $1 \%$ of hydroalcoholic extract of leaves of Pelargonium robertianum $L$. (geranium) was evaluated on the hemorrhoids, in 85 volunteers of both sexes with ages understood between 20 and 50 years, all residents in the city of Lima (Peru). The test, informed previous consent, showed to have effect in the process of to take the soreness out of the hemorrhoids. 74,3\% was evaluated as excellent, 22, $8 \%$ as good and 2,9\% as regulating. During the test allergic reactions were not reported, and the product was very tolerated by the patients. The results newly were observed after the first week of treatment. Due to their natural origin and at their low cost, the present gel can be constituted in a good alternative for the treatment of hemorrhoids.

Key words: Pelargonium robertianum L., hemorrhoids, study clinical, dermatology.

\footnotetext{
* Profesor de la Escuela Académico-Profesional de Farmacia y Bioquímica de la UAP.
} 


\section{INTRODUCCIÓN}

Las hemorroides son venas hinchadas o inflamadas alrededor del ano o al inicio del recto. Son muy comunes, especialmente durante el embarazo y después del parto.

La causa más corriente es el esfuerzo realizado durante la defecación. Sin embargo, el estreñimiento, el permanecer sentado por largos períodos de tiempo y la infección anal son factores que también contribuyen a la aparición de este problema. Las hemorroides internas se presentan donde comienza el recto, mientras que las externas aparecen en el orificio anal, y pueden colgar fuera del mismo, ocasionando molestias y dolor al paciente.

Frente a esta problemática, la medicina natural aporta plantas útiles para su tratamiento, como son las hojas de geranio, las mismas que han venido empleándose desde tiempos inmemoriales para aliviar esta afección.

El presente trabajo intenta darle validez científica a este conocimiento milenario, administrando este recurso natural en forma de gel, con un contenido al $1 \%$ del extracto hidroalcohólico de hojas de Pelargonium robertianum $L$.

\section{MATERIAL Y MÉTODO}

\section{Hipotesis}

El extracto hidroalcóhólico de Pelargonium robertianum $L$., administrado en forma de gel al $1 \%$, tiene efecto antiinflamatorio sobre las hemorroides.

Objetivos

\section{Objetivo general}

Diseñar una forma farmacéutica a base de extracto hidroalcohólico de hojas de geranio para el tratamiento de la hemorroides.

\section{Objetivos especificos}

1. Evaluar el efecto antiinflamatorio del gel al $1 \%$ en el tratamiento de hemorroides.

2. Evaluar la especialidad farmacéutica formulada en cuanto a su estabilidad, compatibilidad química con los excipientes empleados, inocuidad, eficacia, etc.

\section{Metodología}

\section{A. Recolección y preparación del extracto}

Se seleccionaron hojas frescas de Pelargonium robertianum $L$. (geranio) de muestras recolectadas en la ciudad de Lima en el mes de septiembre de 2008 (época de floración).

Se procedió a secarlas en estufa a no más de $40{ }^{\circ} \mathrm{C}$, y el polvo seco fue macerado con alcohol etílico de $70^{\circ}$ por siete días, en oscuridad, para la extracción de los metabolitos. Luego, se filtró y se obtuvo una solución verdosa que se evaporó en estufa de aire circulante, a temperatura no superior a los $40{ }^{\circ} \mathrm{C}$, obteniéndose un extracto pilular.

\section{B. Formulación y estabilidad del gel}

Luego de ensayar varias formulaciones, que no solo fueran compatibles con el principio estudiado sino, sobre todo, estables en el tiempo, se eligió una formulación que cumpliese con estos requisitos y que a la vez tuviese un bajo costo:

Extracto de hidroalcohólico de geranio... 1,00 \% Agentes gelificantes, diluyentes y preservantes $6,90 \%$ Agua destilada estéril c.s.p......................100,00 \%

\section{Ensayo de estabilidad acelerada de la formulación}

Sin duda, un gel bien formulado debe satisfacer diversos criterios, y entre ellos, el poseer 
una buena estabilidad física, porque sin ella, cualquier gel prontamente se vuelve líquido. Por ello, con el fin de determinar la estabilidad del preparado y controlar sus posibles alteraciones, se efectuaron ensayos de estabilidad acelerada, sometiendo la formulación a diferentes temperaturas. Para realizar esta prueba, se procedió a evaluar cada quince días, durante tres meses, los siguientes parámetros: aspecto, consistencia, color, olor y $\mathrm{pH}$.

\section{Ensayo clínico efectuado en voluntarios}

Participaron en el estudio 85 voluntarios sanos de ambos sexos, con edades comprendidas entre 20 y 50 años, residentes en diversos distritos de Lima Metropolitana.
Se dividió a los participantes en dos grupos: Grupo I (grupo problema, de 70 pacientes), al que se administró gel con extracto de geranio, y Grupo II (grupo control, de 15 pacientes), al que se administró gel sin extracto de geranio.

Previo consentimiento escrito, a los pacientes con hemorroides se les aplicó el gel a base de geranio dos veces al día, previo lavado con jabón de glicerina.

El estudio se extendió durante 30 días, durante los cuales se administró una dosis de preparado de aproximadamente 3,0 g diarios, repartidos en dos dosis cada 12 horas, excluyendo la utilización de cualquier crema convencional. El seguimiento clínico se realizó cada 7 días.

\section{RESULTADOS}

\section{A. Evaluación farmacotécnica del gel}

\section{FICHA TÉCNICA DE ESTABILIDAD DEL PRODUCTO TERMINADO}

$\begin{array}{lll}\text { PRODUCTO } & : & \text { Extracto de geranio } \\ \text { FORMA COSMET. } & : & \text { Gel } \\ \text { PRESENTACIÓN } & : & \text { Frasco x } 50 \mathrm{~g} \\ \text { N }^{\circ} \text { DE LOTE } & : & \text { Piloto N } 001 \\ \text { FECHA FAB. } & : & \text { Octubre 2008 } \\ \text { FECHA VENC. } & : & \text { Octubre 2011 }\end{array}$

\begin{tabular}{|c|c|c|c|c|c|}
\hline \multirow{2}{*}{ DETERMINACIONES } & \multirow{2}{*}{ ESPECIFICACIONES } & \multicolumn{4}{|c|}{ RESULTADOS } \\
\hline & & Inicio & $1 \mathrm{mes}$ & 2 meses & 3 meses \\
\hline ASPECTO & $\begin{array}{l}\text { Gel opaco sin } \\
\text { burbujas ni impurezas } \\
\text { visibles. }\end{array}$ & Cumple & Cumple & Cumple & Cumple \\
\hline COLOR & Verde & Cumple & Cumple & Cumple & Cumple \\
\hline OLOR & Sui géneris & Cumple & Cumple & Cumple & Cumple \\
\hline VISCOSIDAD & $20000-30000 \mathrm{cps}$ & 20000 & 21000 & 21000 & 21000 \\
\hline $\mathrm{pH}$ & $6,0-7,5$ & 6,8 & 6,8 & 6,8 & 6,8 \\
\hline \multicolumn{6}{|l|}{ RECUENTO MICROBIANO } \\
\hline AEROBIOS MESÓFILOS & Menos de $100 \mathrm{ufc} / \mathrm{g}$ & Conforme & Conforme & Conforme & Conforme \\
\hline RECUENTO DE HONGOS & Menos de $10 \mathrm{ufc} / \mathrm{g}$ & Conforme & Conforme & Conforme & Conforme \\
\hline RECUENTO DE LEVADURAS & Menos de $10 \mathrm{ufc} / \mathrm{g}$ & Conforme & Conforme & Conforme & Conforme \\
\hline ESCHERICHIA COLI & Ausente en $10 \mathrm{~g}$ & Conforme & Conforme & Conforme & Conforme \\
\hline SALMONELLA spp. & Ausente en $10 \mathrm{~g}$ & Conforme & Conforme & Conforme & Conforme \\
\hline $\begin{array}{l}\text { STAPHYLOCOCCUS } \\
\text { AUREUS }\end{array}$ & Ausente en $10 \mathrm{~g}$ & Conforme & Conforme & Conforme & Conforme \\
\hline $\begin{array}{l}\text { PSEUDOMONAS } \\
\text { AERUGINOSA }\end{array}$ & Ausente en $10 \mathrm{~g}$ & Conforme & Conforme & Conforme & Conforme \\
\hline
\end{tabular}




\section{B. Evaluación clínica}

Los resultados del comportamiento clínico a la $1^{\text {a }}, 2^{\text {da }}, 3^{\text {era }}$ y $4^{\text {ta }}$ semana de tratamiento se aprecian en la tabla 2. Nótese que a la primera semana de aplicación del gel, ningún paciente del grupo I manifestó apreciable mejoría, lo que sí se evidenció notoriamente a partir de la segunda semana. De otro lado, la totalidad de pacientes del GRUPO II (grupo control) no experimentó efectos durante todo el ensayo.

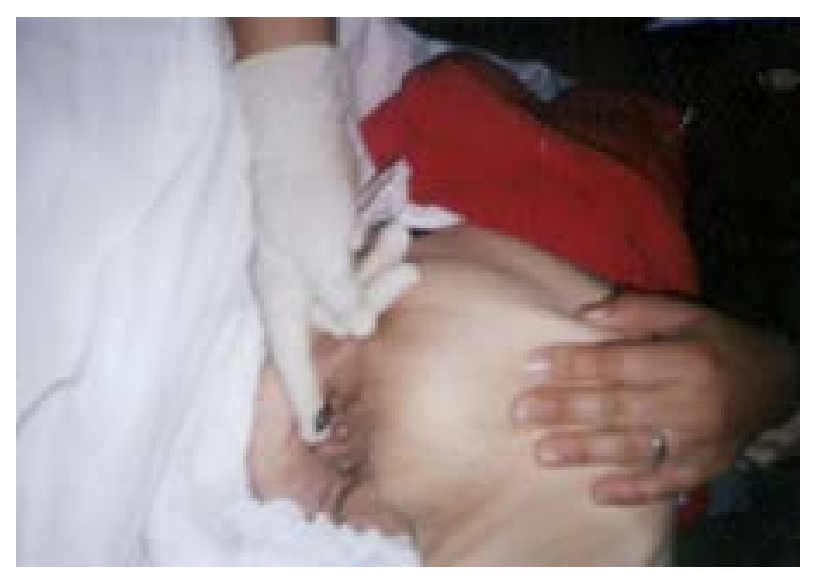

Tabla 2. Valoración clínica por semanas

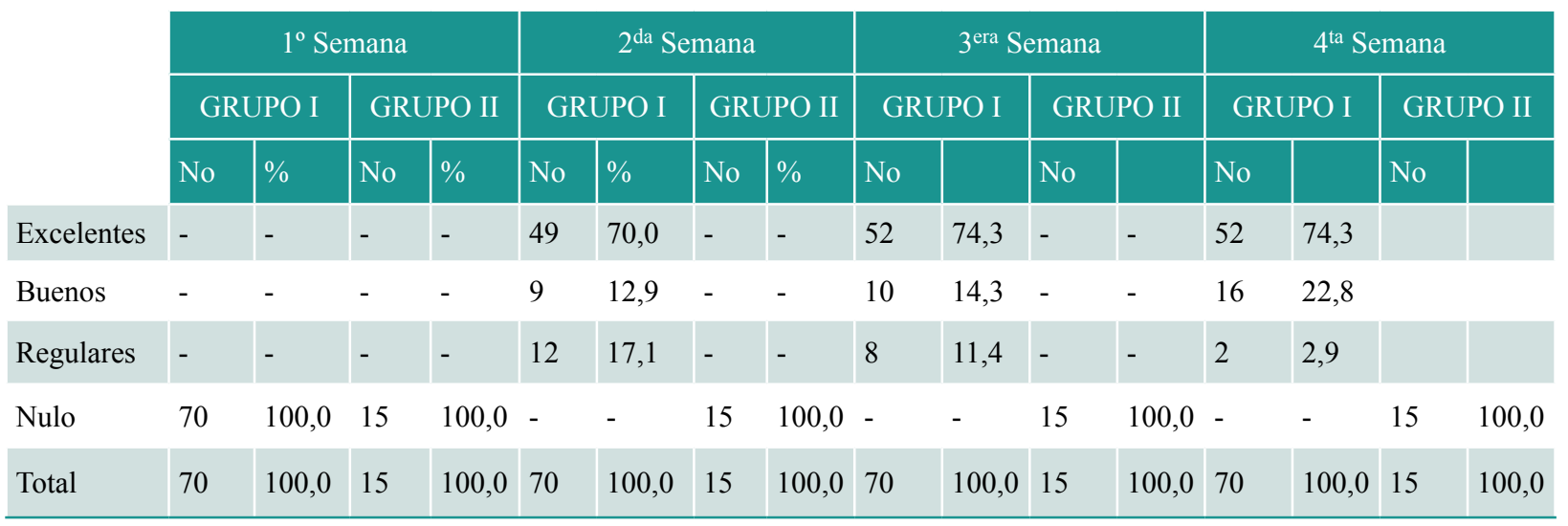

En consecuencia, de acuerdo con la tabla calificadora de resultados de Balbuena Gonzales y Chapano Jairo (1987), la evaluación clínica corresponde a la clasificación que se señala en la tabla 3.

\section{Tabla 3}

\begin{tabular}{|l|c|c|}
\multicolumn{1}{|c|}{ CALIFICACIÓN } & $\begin{array}{c}\mathrm{N}^{\circ} \\
\text { casos }\end{array}$ & $\%$ \\
\hline $\begin{array}{l}\text { EXCELENTES } \\
\text { (reducción entre 80-100\%) }\end{array}$ & 52 & 74,3 \\
\hline $\begin{array}{l}\text { BUENOS } \\
\text { (reducción entre el 60-80 \%) }\end{array}$ & 16 & 22,8 \\
\hline $\begin{array}{l}\text { REGULARES } \\
\text { (reducción entre 40-60 \%) }\end{array}$ & 2 & 2,9 \\
\hline $\begin{array}{l}\text { NULO } \\
\text { (reducción menor de un 40\%) }\end{array}$ & - & - \\
\hline SUBTOTAL & 70 & 100 \\
\hline GRUPO CONTROL & 15 & \\
\hline TOTALES & 85 & \\
\hline
\end{tabular}
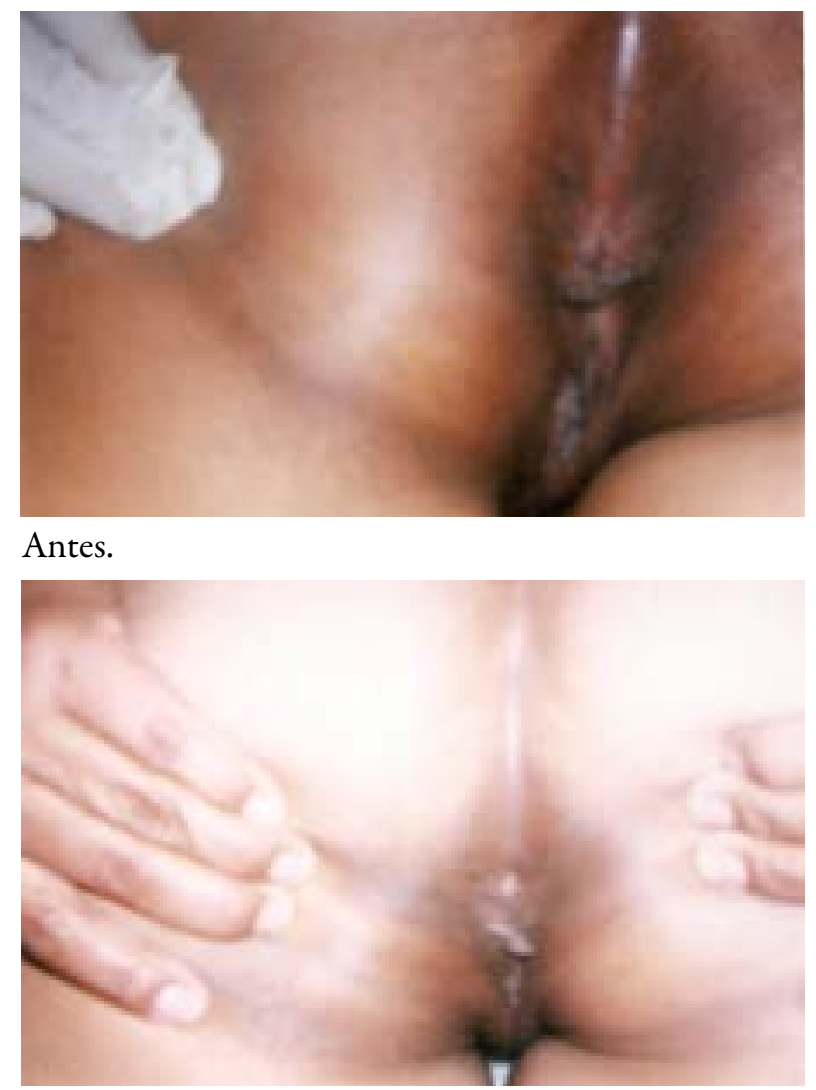

Después. 


\section{ANÁLISIS Y DISCUSIÓN}

Los resultados obtenidos muestran una marcada acción favorable de parte del gel formulado al $1 \%$ de extracto hidroalcohólico de Pelargonium robertianum $L$. (geranio) en el proceso desinflamatorio de las hemorroides (fig. $1 \mathrm{y}$ tablas 2 y 3$)$.

Durante la evaluación semanal, se observó que durante la primera semana de tratamiento las hemorroides no evidenciaron mejoría, lo que desanimó un poco a los investigadores y a los propios pacientes. Sin embargo, a la segunda semana, los resultados fueron asombrosos; las hemorroides comenzaron a disminuir notoriamente, progresando hasta la tercera semana. Durante la cuarta semana, los progresos fueron mínimos en razón de que el 74,3 \% estaba ya totalmente desinflamado.

Los participantes en el estudio renunciaron a cualquier tratamiento oral o tópico durante el ensayo, y mostraron su total complacencia por los resultados. Así, se puede decir, en términos generales, que el estudio tuvo una evolución satisfactoria.

Se puede concluir que la rutina y la quercetina, verdaderos inhibidores de la enzima ciclooxigenasa, serían las principales responsables del efecto ensayado, además, indudablemente, de los demás componentes que sinergizan la acción de este importante fitocomplejo.

Finalmente, puede afirmarse que la formulación ensayada mostró gran estabilidad física y química, y respondió satisfactoriamente a las pruebas de estabilidad acelerada, alcanzando una vigencia de 3 años, razón por la que estaríamos frente a un producto farmacéutico efectivo y de bajo costo para el tratamiento de las hemorroides.

\section{CONCLUSIONES}

1. El extracto hidroalcohólico de Pelargonium robertianum L., administrado bajo la forma de gel al 1\%, mostró tener buen efecto en el tratamiento de las hemorroides.

2. Por su fácil adquisición, bajo costo y ser una sustancia de origen natural que ha demostrado tener efecto sobre las hemorroides, el gel de geranio al $1 \%$ puede constituir un importante activo para aplicación en dermatología.

\section{REFERENCIAS BIBLIOGRÁFICAS}

1. Bézanger-Beauquesne, L; Pinkas, M; Torck, M. Les Plantes dans la Therapeutique Moderne. Paris, Maloine, 1986, pp.320-1.

2. British Herbal Pharmacopoeia. Vol. I. Bournemouth, Dorset, British Herbal Medical Association, 1990, pp. 69-70.

3. D'Arcy, PF. Adverse reactions and interactions with herbal medicines. Part II. Drug interactions. Adverse Drug React Toxicol Rev, 1993; 12 (3): 147-162.

4. Fernández, M; Nieto, A. Plantas medicinales. Pamplona, Ediciones Universidad de Navarra, 1982, p. 130.

5. Le Floc'h, E. Contribution a une Etude Ethnobotanique de la Flore Tunisienne. Imprimerie Officielle de la Republique Tunisienne, 1983, p. 177.

6. Paris, RR; Moyse, M. Précis de Matiére Médicale. Tome II. Paris, Masson, 1967, pp. 475-6.

7. Van Hellemont, J. Compendium de Phytotherapie. Bruxelles, Association Pharmaceutique Belge, 1986, pp. 38-40.

8. Wichtl, M. Herbal Drugs and Phytopharmaceutical. A Handbook for Practice on a Scientific basis. Stuttgart, Medpharm Scientific Publishers, 1994, pp. 369-72.

9. http://lashierbasdelaabuela.blogspot.com

10. http://www.fitoterapia.net/vademecum/index.html 